\title{
Semianalytical Description of Nonlocal Secondary Electrons in a Radio Frequency Capacitively Coupled Plasma at Intermediate Pressures
}

\author{
Stanislav V. Berezhnoi, Igor D. Kaganovich, Martin Mišina, Annemie Bogaerts, and Renaat Gijbels
}

\begin{abstract}
A model for fast simulation of a radio-frequency capacitively coupled discharge in the pressure range of a few torr and higher has been developed. The semi-analytical description of the nonlocal ionization by secondary $(\gamma)$ electrons allows us to evaluate the discharge parameters in detail even when the local approach for $\gamma$ electrons is not valid. We focused on the effect of steep increase of the plasma density at the central part of the discharge, at the transition from $\alpha$ to $\gamma$ regime. The present study demonstrates that in the $\gamma$ regime the plasma density at the discharge center is proportional to the spatial scale of ionization decay by $\gamma$ electrons inside the plasma region. We have investigated the electron energy distribution function of $\gamma$ electrons both analytically and numerically. As a result, the analytical formulas for the spatial scales of ionization decay by $\gamma$ electrons were found in limiting cases.
\end{abstract}

Index Terms-Nonlocal ionization by secondary electrons, radio frequency capacitively coupled discharge.

\section{INTRODUCTION}

$\mathbf{S}$ TEADY interest in radio-frequency capacitively coupled (RFC) discharges has existed for a long time due to the application of these discharges in plasma processing, laser technology, analytical chemistry, etc.

RFC discharges at intermediate pressures were investigated at length (see, for instance, [1]). It is known that at high voltages, ionization by $\gamma$ electrons exceeds the ionization by electrons generated in the plasma. Moreover, a steep increase of the plasma density at the discharge center occurs with increasing voltage. In [2] it was supposed that the reason of

Manuscript received December 11, 1998; revised May 14, 1999. This work was funded by the University of Antwerp, Belgium (New Research Initiative "Mathematical Simulation of Pulsed, Radio-Frequency and Magnetron Discharges.") The work of S. V. Berezhnoi was supported in part by INTAS, Grant 96-0235, by the Russian Foundation for Basic Research, Project 9802-16000, and by a personal grant for young scientists in the plasma physics field "UTS i plasmennye processy" N378. The work of I. D. Kaganovich and M. Mišina was sponsored by the Belgian Office for Scientific Technical and Cultural Affairs, Scientific and Technical collaboration with Central and Eastern Europe. The work of I. D. Kaganovich was also partially supported by the National Science Foundation, CTS-9713262. The work of A. Bogaerts was supported by the Flemish Fund for Scientific Research (FWO).

S. V. Berezhnoi is with the Physical Technical Department, St. Petersburg State Technical University, 195251, Russia (e-mail: berezhnoj@phtf.stu.neva.ru).

I. D. Kaganovich is with the Department of Chemical Engineering, University of Houston, Houston, TX 77204-4792 USA (e-mail: ikaganov@jetson.uh.edu).

M. Mišina, A. Bogaerts, and R. Gijbels are with the Department of Chemistry, University of Antwerp, Belgium. (e-mail: misina@fzu.cz; bogaerts@uia.ua.ac.be; gijbels@uia.ua.ac.be).

Publisher Item Identifier S 0093-3813(99)08119-9. that increase is the nonlocality of $\gamma$ electrons. However, the influence of nonlocality of secondary electrons on the plasma density growth in RFC discharges has never been investigated numerically. This paper is devoted to a numerical investigation of this effect.

Modeling of RFC discharges have commonly been performed in two ways, namely - by solving the ion continuity equation with 1) the ionization calculated by use of the Monte Carlo collisions (MCC) technique for electrons see, e.g., [3], and 2) by utilizing the Townsend approximation for ionization [1]. MCC collision techniques are very computationally intensive. This disadvantage creates a substantial barrier for the investigation of plasma profiles, since the smallest time scale involved in the computation $(\gamma$ electrons pass through the sheath region in $10^{-9} \mathrm{~s}$ ) is $10^{6}$ times less than the time evolution of the plasma profile associated with ion diffusion through the bulk plasma $\left(10^{-3} \mathrm{~s}^{-1}\right)$. The Townsend approximation is based on the assumption that the ionization frequency can be parameterized only by the value of the local electric field. As it was pointed out in paper [13], the sheath width in the $\gamma$ regime of dc and RFC discharges automatically shrinks to a small value which is comparable with the relaxation length of $\gamma$ electrons even at high pressures. This implies that such an approximation is not valid for any discharge pressure.

The goal of the present paper is to generalize the Townsend approximation to include nonlocal effects (dependence on nonlocal electric field) by simple approximate formulae.

To do so we shall introduce a number of approximations, most of them are justified by corresponding small parameters, and some are justified by comparison with results of MCC calculations. The accuracy of the approximation for nonlocal ionization is in the range of 30-50\%, but these approximations lead to a giant speed up of calculations, compared with MCC methods, which are caused by a factor of $10^{6}$ difference in time scales.

The procedure of calculation is described in Section II. Section III contains the results of modeling and their comparison to the experimental data [1]. We have shown that the variation of the spatial scale of the decay of ionization induced by $\gamma$ electrons causes a strong variation of the plasma density profile at the discharge center. An explanation of this phenomenon is given in Section IV. The analytical solution for the electron energy distribution function of $\gamma$ electrons is presented in Section V. By comparison of the analytical 
formula with MCC numerical results the analytical expression for nonlocal ionization is verified. The last section contains the conclusions.

\section{The Full Self-Consistent System OF EQUATIONS FOR FAST MODELING OF AN RFC DISCHARGE AT INTERMEDIATE PRESSURES}

We performed self consistent calculations of RFC discharges over a wide range of pressures, and power densities according to the fast modeling techniques, developed in [5] and [6].

The choice of the numerical techniques we used is stipulated by several reasons. First of all, fast modeling techniques allowed us to investigate a wide range of controlled variables. Second, we were able to single out important physical processes, responsible for plasma profile formation. As discussed above the disparities in time and space scales are serious barriers to physical investigation by "brute force" approaches. For example, the typical time for an ion to pass through the sheath is about $10^{-6} \mathrm{~s}$, whereas bulk profiles evolve for $10^{-3} \mathrm{~s}$. It implies the necessity of at least 1000 iterations to reach steady state. Without eliminating electron time scales this problem requires tremendous computational efforts. Since our focus was to study mechanisms responsible for the abrupt increase of the plasma central density during $\alpha-\gamma$ transition, we ultimately needed to utilize fast modeling techniques (FMT).

However, a number of initial assumptions are necessary to validate FMT. The approximations have been discussed in detail in [5] and [6], and we reproduce them briefly below.

The discharge is separated into a plasma and a sheath region. During the plasma phase bulk electrons are present in the sheath, during the phase of ionic space charge, the electron density of bulk electrons is nearly zero; the net positive charge generates a high electric field. The width of the transition region between the two phases is of the order of the Debye radius and is treated as infinitely thin, based on the assumption that $U \gg T_{e} / e$, where $U$ is the discharge voltage, $T_{e}$ is the electron temperature.

The electric field in different regions is calculated separately. In the plasma the time averaged field corresponds to the absence of any dc electron current and leads to ambipolar plasma diffusion. The time dependent RF electric field can be found from the law of current conservation

$$
j=j_{e}+j_{i}+j_{d}=\sigma_{e} E+\sigma_{i} E+(1 / 4 \pi)(\partial E / \partial t) .
$$

In the plasma the displacement current $j_{d}$ and the ion current $j_{i}$ are negligible in comparison with the electron current $j_{e}$, assuming

$$
\sigma_{i} \ll \omega /(4 \pi) \ll \sigma_{e}
$$

where $\sigma_{i}, \sigma_{e}$ are the ion and electron conductivity, respectively, and $\omega$ is the discharge frequency.

In the ionic space charge region, where and when electrons are not present spatially and temporally, $j=j_{d}$. We consider a current density $j=j_{0} \sin (\omega t)$ as an external parameter instead of the typically used voltage. Integrating current conservation with zero electric field at the edge of the ionic space charge (ISC), the electric field reads

$$
E_{I S C}(x, t)=\frac{4 \pi j_{0}}{\omega}[\cos (\omega t) \mp \cos (Z)]
$$

where $Z=Z(x)$ is the inverse function of $x(Z)$-the edge position of the ionic space charge at the RF field phase $Z=\omega t$, "-"corresponds to the left sheath, "+" to the right sheath. The function $Z(x)$ can be found by substituting the electric field (3) into the Poisson equation: $\sin (Z)(d Z / d x)=e\left(\omega / j_{0}\right) n(x)$, $Z=0$ at the plasma sheath boundary, $Z= \pm \pi$ at the discharge surface.

The density profile is to be found from the ion continuity equation. We assume that the ion response time on variation of the electric field in the sheath is large compared with the discharge current period, so that the ion profile is quasistationary. This assumption is restricted by the left condition (2), and is not valid for very high currents.

The ion continuity equation averaged over the RF period can be used [5], [6]

$$
\frac{d}{d x}\langle\Gamma\rangle=\langle I\rangle
$$

where $\langle\Gamma\rangle$ is time-averaged ion flux, and $\langle I\rangle$ is time averaged ionization rate. Under the conditions that half of the discharge gap $L$ and the sheath thickness $L_{s h}$ exceed the ion mean free paths, ion motion can be described by the drift-diffusion approximation

$$
\langle\Gamma\rangle=\mu_{i}\left\langle E_{I S C}\right\rangle n-D_{a m b}\left(1-\frac{Z}{\pi}\right) \frac{\partial n}{\partial x} .
$$

$D_{a m b}=-\mu_{i} T_{e} / e$ is the ambipolar diffusion coefficient. The boundary conditions are $d n /\left.d x\right|_{x=0}=0$ at the plasma center, and $d\langle\Gamma\rangle /\left.d x\right|_{x=L}=0$.

The ionization rate is traditionally separated into two parts $\left\langle I_{\alpha}\right\rangle+\left\langle I_{\gamma}\right\rangle$, where $\left\langle I_{\alpha}\right\rangle,\left\langle I_{\gamma}\right\rangle$ represent ionization, averaged over the RF period, by plasma bulk electrons and high energy electrons, created by secondary electron emission at the walls and accelerated in high electric fields in ISC regions.

In the considered pressure range, the following inequalities are valid $\omega \ll \nu * \ll \nu$, where $\omega$ is the discharge frequency, $\nu *$ is the excitation frequency, and $\nu$ is the elastic collision frequency; $L_{s h}$ exceeds ion and the electron mean free paths, and electron energy relaxation length $\lambda^{*} \ll L_{s h}, L$. Under these assumptions, the electron distribution function (EDF) depends only on the instantaneous local electric field.

So, we can use the Townsend approximation to find the ionization rate instead of solving the electron kinetic equation [1]. The Townsend approximation for $\alpha$ ionization can be written as

$$
\begin{aligned}
I_{\alpha}(x, t) & =\alpha(x, t) \Gamma_{e}(x, t) \\
& =n_{e} \mu_{e}\left|E_{p l}(x, t)\right| \alpha\left(\left|E_{p l}(x, t)\right| / N_{a}\right)
\end{aligned}
$$

where $\alpha$ is an experimentally or numerically obtained function which is defined by gas properties, $e$ is the electron flux, and the electric field $E_{p l}$ is given by (1), which in the plasma simplifies to $j_{0} \sin (\omega t)=\sigma_{e} E_{p l}$; in a given pressure range the main part (body) of the EDF is quasi-stationary $(\omega>2 \mathrm{~m} / M \nu)$ 
so that the conductivity does not depend on time [7]. The ionization by gamma electrons is governed by the flux of $\gamma$ electrons $I_{\gamma}(x, t)=\alpha\left(E_{I S C}(x, t)\right) \Gamma_{e \gamma}(x, t), \gamma$ electrons move quickly through the sheath toward the plasma and their flux increases. Integrating the flux starting from the electrode results in exponential flux multiplication [6]

$$
\begin{aligned}
I_{\gamma}(x, t)= & \alpha\left(E_{I S C}(x, t)\right) \gamma \Gamma_{i}(L)(1+\cos (\omega t)) \\
& \cdot \exp \left[\int_{x}^{L} \alpha\left(E_{I S C}\left(x^{\prime}, t\right)\right) d x^{\prime}\right]
\end{aligned}
$$

where $\gamma$ is the coefficient of secondary ion-electron emission, the coefficient $\alpha$ is a function of the electric field of the ionic space charge (3). Equation (6) utilizes a local approximation for $\gamma$ electrons. The typical relaxation length of high energy electrons $(\varepsilon \sim 100 \mathrm{eV})$ is several inelastic mean free paths and is much larger than the relaxation length of the EDF tail of $\alpha$ electrons which is one mean free path, so that the local approximation may be valid for $\alpha$ electrons and not valid for $\gamma$ electrons. As we shall see, nonlocal effects invoke a number of physical phenomena. We shall discuss these phenomena in Section IV.

Under the conditions used for the calculations, the neutral gas heating results in an increase of the neutral gas temperature $T(x)$ and leads to an inhomogeneous neutral gas density. Since the gas pressure is uniform $k N_{a}(x) T(x)=p=$ const, it must be accounted for in the calculations of ionization and mobilities. The neutral gas temperature is to be found from the heat conductivity equation

$$
\frac{d}{d x} \Lambda(T) \frac{d T}{d x}+\left\langle E_{p l} j\right\rangle=0
$$

where $\left\langle E_{p l} j\right\rangle$ is the time-averaged Ohmic heating in the plasma phase, $\Lambda(T)=\Lambda_{0} \sqrt{T / T_{0}}, \Lambda_{0}$ is the thermal conductivity at room temperature $T_{0}=300 \mathrm{~K}$. The boundary conditions are $T(L)=T_{0}, d T /\left.d x\right|_{x=0}=0$ (it was assumed that the electrodes are cooled effectively).

Thus, the system of equations is complete. It consists of time-averaged ion continuity (4), Poisson equation for $Z(x)$ and heat conductivity (7). Time-averaging of the ionization rate was performed numerically. The system of equations is highly nonlinear, so that it was solved by iterations (as steady state of a time dependent problem, similar to [8]).

This set of equations was solved extensively in [9] for an investigation of current-voltage characteristics of RFC discharges in a number of inert gases at higher pressures $\sim 100$ torr. ${ }^{1}$

\section{RESUlts OF MODELING AND COMPARISON WITH EXPERIMENTAL DATA}

The experimental data for comparison with our simulation results were taken from [4] where extensive investigation of plasma parameters of RFC discharges was performed. The

\footnotetext{
${ }^{1}$ Under the considered pressure range the electron-ion recombination is not important unlike [6], [9]. Actually recombination is important if $\mathrm{n}>\sim$ $\left(D_{a b m} / L_{p}^{2} \beta\right)$, where $\beta$ is the coefficient of the electron-ion recombination, and $L_{p}=L-L_{s h}$. When the background gas is argon at $p=3$ torr, $L_{p} \approx 3$ $\mathrm{cm}, D_{a b m} \approx 10^{4} \mathrm{~cm}^{2} \mathrm{~s}^{-1}$, and $\beta \approx 10^{11} \mathrm{~cm}^{-3} \mathrm{~s}^{-1}$ [12] $n$ must exceed the value $\sim 10^{13-14} \mathrm{~cm}^{3}$, which is far beyond the explored range of densities
}

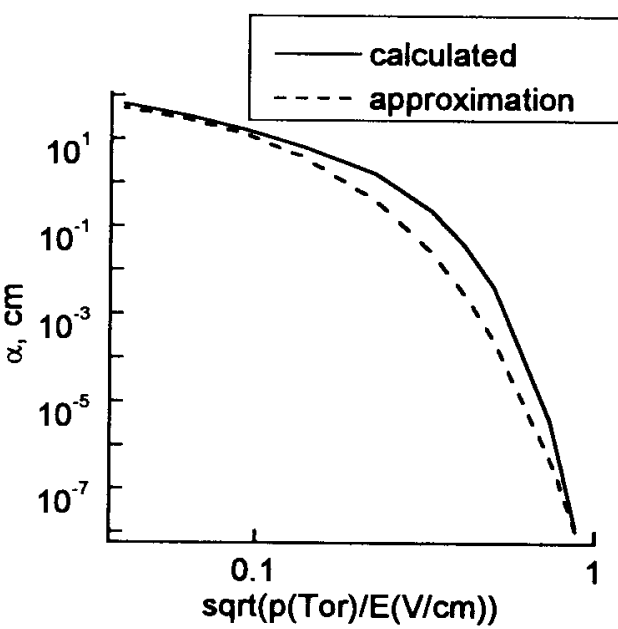

(a)

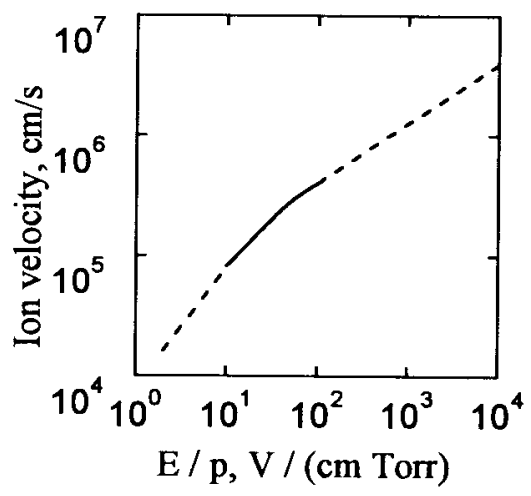

(b)

Fig. 1. (a) Numerically obtained Townsend coefficient for ionization of neutral atoms of argon (solid line) compared to exponential approximation $\alpha=C p \exp \left(-\sqrt{\left(G^{2} p\right) /|E|}\right) C=29.2 \mathrm{~cm}^{-1}$ torr $^{-1}, G=26.6(\mathrm{~V} /(\mathrm{cm}$ torr) $)^{-1 / 2}$ from [10] (dashed line) and (b) ion velocity as a function of electric field from [10].

discharge parameters are: frequency $\omega=13.56 * 2 \pi \mathrm{MHz}$, half of the gap $L=3.35 \mathrm{~cm}$, argon pressure $p=3$ torr. The constants which were used for the calculations are: electron mobility $\mu_{e}=0.43 \times 10^{2} \mathrm{~m}^{2} / \mathrm{V} / \mathrm{s}$ (at 1 torr), gas heat conductivity $\lambda_{0}=10^{4} \mathrm{~W} /(\mathrm{cm} \mathrm{K}), T_{0}=300 \mathrm{~K}$.

For discharges in helium and argon, the Townsend coefficient is typically approximated in the form

$$
\alpha=\frac{C p T_{0}}{T} \exp \left(-\sqrt{\frac{G^{2} p T_{0}}{|E| T}}\right)
$$

where $C, G$ are constants [1]. In the given form the approximation of the Townsend ionization coefficient is appropriate at high $(E / p)$ ratio. However, we found that this approximation fails at low $E / N_{a}$, and is invalid for the description of ionization at the discharge center, where the electric field is low. So, the electron Boltzmann kinetic equation [7] was solved for a series of $E / N_{a}$ values to obtain $\alpha$. The results of the calculations are shown in Fig. 1(a). The dependency of the ion mobility on $E / p$ was taken from [7] [see Fig. 1(b)], and the coefficient of secondary ion-electron emission was assumed to be equal to 0.1 . 


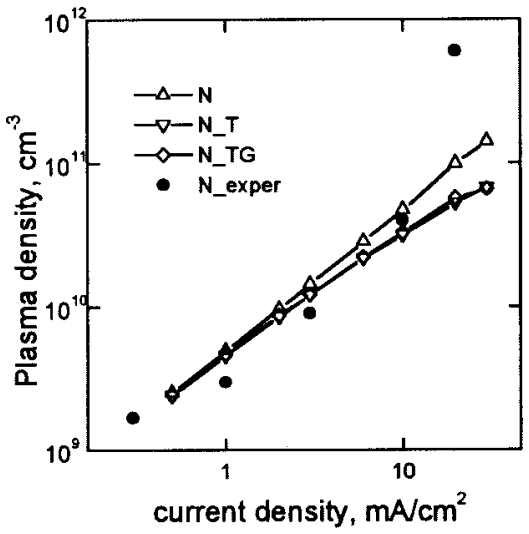

(a)

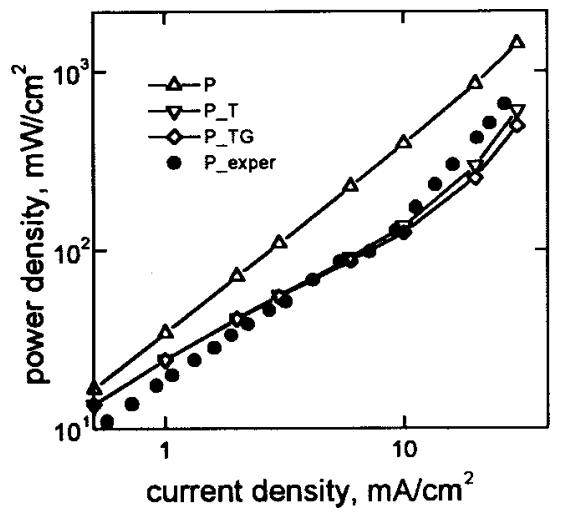

(b)

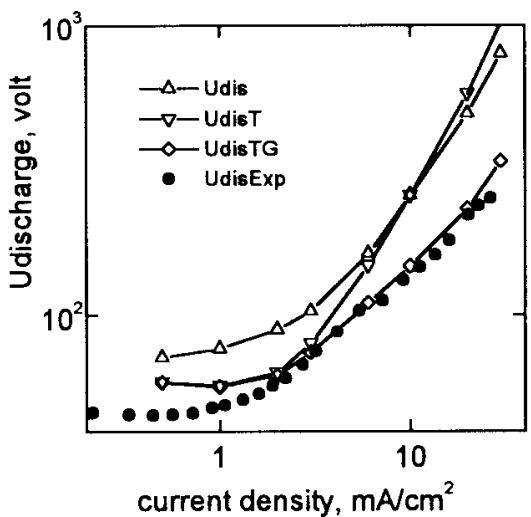

(c)

Fig. 2. Calculated parameters of RFC discharge. The parameters are: $\omega=13.562 \pi \mathrm{MHz}, L=3.35 \mathrm{~cm}$, argon $p=3$ torr. (a) The calculated plasma density at the discharge center, (b) power per unit area, and (c) discharge voltage versus current density are presented in comparison with the experimental data. The experimental data are represented by dots. " $T$ " marks the results of calculations performed taking neutral gas heating into account. "TG" marks the results of calculations performed taking neutral gas heating and gamma ionization into account.

In Fig. 2, the resulting plasma density at the discharge center, power density, and discharge voltage are presented in comparison with the experimental data. The various series of calculations were performed: 1) without taking into consideration neutral gas heating and $\gamma$ ionization, 2) with taking into consideration neutral gas heating (the corresponding results in Fig. 2 are marked by "T"), and 3) accounting for $\gamma$ ionization and neutral gas heating (the corresponding results are marked

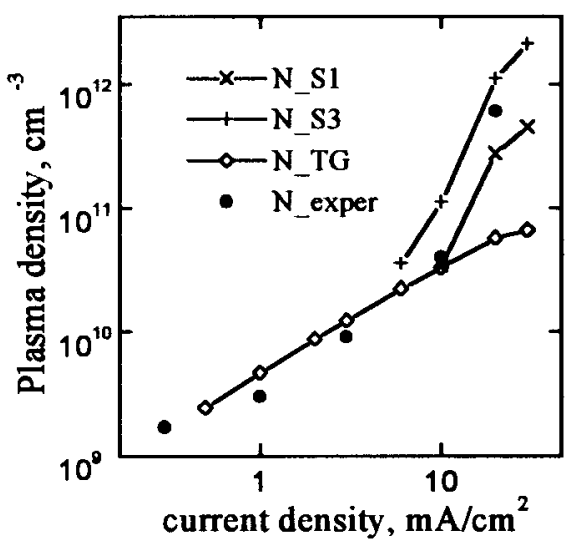

(a)

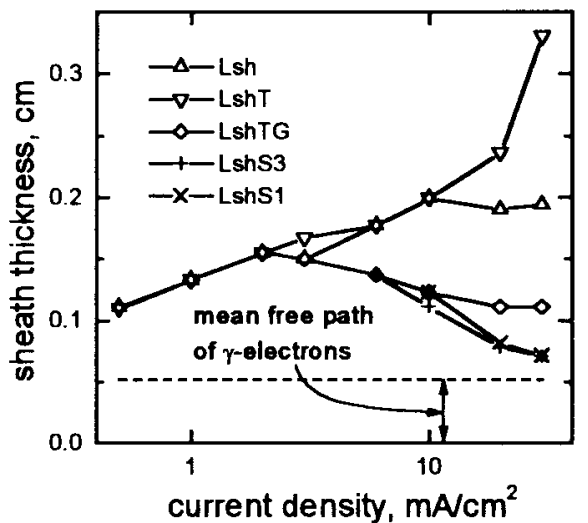

(b)

Fig. 3. (a) The resulting plasma density at the discharge center and (b) sheath thickness are presented versus current density. The results corresponding to $k=1,3$ (see text) are represented by curves marked by "S1," "S3," respectively.

by "TG"). The power density is a sum of power deposited in electrons and ions $\int_{0}^{L}\left(\left\langle j_{e} E\right\rangle+\left\langle j_{i} E\right\rangle\right) d x$.

We can see that simulation results are consistent with experimental data when gas heating and $\gamma$ ionization are accounted for. Discrepancies in the discharge voltage exist at low currents below $1 \mathrm{~mA} / \mathrm{cm}^{2}$ and in the central plasma density at large currents. The possible cause for a higher calculated voltage than the experimental one is the stepwise ionization of metastable atoms which was not incorporated in the model. Inclusion of ionization of metastable atoms into the model is rather cumbersome, since it requires recalculation of the Townsend coefficient. The electron excitation and ionization of metastables influence EDF [11], so that a correct model for the Townsend coefficient should include dependence on the metastable atom density as well. The contribution of the metastable atoms to the ionization is negligible at higher current densities [12].

The second disagreement is associated with the inadequate description of $\gamma$ ionization. The results of the calculations do not show any steep increase of plasma density growth at the discharge center at high voltages unlike the experimental data [see Fig. 3(a)]. The reason for such an increase is the nonlocality of ionization by $\gamma$ electrons [2]. The $\gamma$ ionization exceeds the ionization by the electrons generated in the plasma 
phase in the sheath at high current densities. If ionization by $\gamma$ electrons is spread into the plasma region it leads to a steep increase of ionization near the plasma-sheath boundary. Consequently, the plasma density at the discharge center increases too. To check this fact, calculations were performed with a spread $\gamma$ ionization. Spreading was performed according to

$$
I_{\text {nonlocal }}(x)=\int_{x}^{L} I_{\gamma}(y) \exp \left(-(y-x) / k \lambda_{\gamma}\right) d y / k \lambda_{\gamma}
$$

where $\lambda_{\gamma}$ is the mean free path of $\gamma$ electrons for ionization at energy $40 \mathrm{eV}, k$ is some constant determining the depth of spreading. Calculations were performed for $k=1,3$. Equation (8) is a very simplified method to account for nonlocality. There are two reasons for its utilization. It gives results very close to MCC simulation results, and it is the most simple way of calculation. A similar estimate was proposed in [4]. We have postponed the detailed discussion until Section V. The resulting plasma density at the discharge center is shown in Fig. 3(a) as a function of the current density. The sheath thickness is presented in Fig. 3(b). The data corresponding to $k=1,3$ are represented by curves marked by "S1," "S3," respectively. The discharge voltage varied insignificantly, that is why no data are shown.

The results of the calculations demonstrate that taking into account the nonlocality of $\gamma$ electrons causes a dramatic increase of plasma density. Qualitatively, the more the spreading of $\gamma$ ionization, the higher is the plasma density.

In Fig. 4 the profiles of (a) plasma density, (b) ionization, and (c) neutral gas temperature are shown for $k=1,3$ and $k=0$, (i.e., without accounting for nonlocality) at $j_{0}=20$ $\mathrm{mA} / \mathrm{cm}^{2}$, and for $k=0$ at $j_{0}=2 \mathrm{~mA} / \mathrm{cm}^{2}$.

The ion density profile is nonmonotonic for the case in which nonlocality at high currents was not accounted for [for instance, see Fig. 4(a), $j_{0}=20 \mathrm{~mA} / \mathrm{cm}^{2}$ ]. This is connected to the fact that at high voltages the ion ionization rate has a sharp maximum inside the sheath. When nonlocality of $\gamma$ ionization is accounted for, the maximum of the ion source moves inside and the ion flux does not grow inside the sheath. As a result, the ion density peak disappears.

The nonlocality of ionization leads to a significant ion density growth in the plasma in the $\gamma$ regime. Consequently, the electric field diminishes (it is inversely proportional to density) decreasing the gas heating and the temperature.

\section{INFLUENCE OF NONLOCAL IONIZATION PROFILES on Plasma Density in the Discharge BulK}

The influence of nonlocal ionization profiles on the plasma density in the discharge bulk can be estimated analytically. Consider (4) in the plasma region at $|x|<L_{p}$

$$
-\frac{d}{d x} D_{a m b} \frac{d n}{d x}=\sum\left\langle I_{\alpha}\right\rangle+\left\langle I_{\beta}\right\rangle .
$$

The boundary condition for (8) at the sheath can be written as a generalized Bohm criterion [6] $-D_{a m b}(d n / d x)=V(\delta) n$, where $\delta$ is of the order of the Debye radius and has to satisfy equation $V(\delta), \delta=D$ by definition [6], $V(x)$ is the ion velocity in the sheath. This boundary condition corresponds

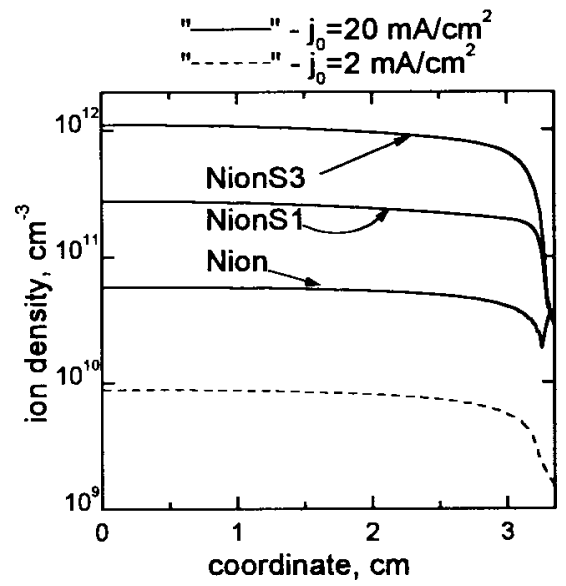

(a)

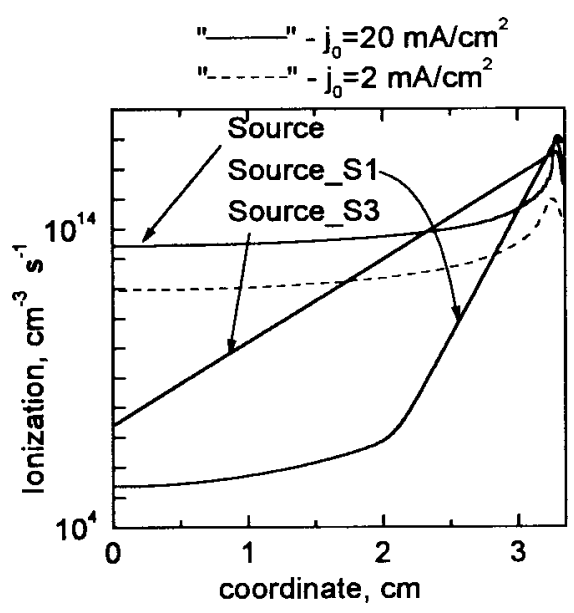

(b)

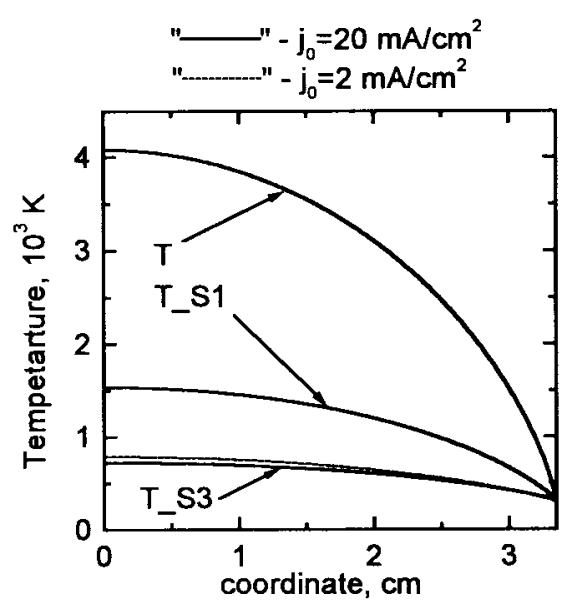

(c)

Fig. 4. (a) The profiles of plasma density, (b) ionization, and (c) neutral gas temperature are shown for $k=1,3$ and $k=0$ (without considering nonlocality) at $j_{0}=20 \mathrm{~mA} / \mathrm{cm}^{2}$ (solid lines), and for $k=0$ at $j_{0}=2$ $\mathrm{mA} / \mathrm{cm}^{2}$ (dashed lines). The results corresponding to $k=1,3$ (see text) are represented by curves marked by "S1," "S3," respectively.

to the transition from the diffusion-dominated flux to the convective-dominated flux in the sheath.

At high current densities the $\gamma$ ionization considerably (by several orders of magnitudes) prevails over the ionization by plasma electrons; see Fig. 4(b). When the nonlocality of 


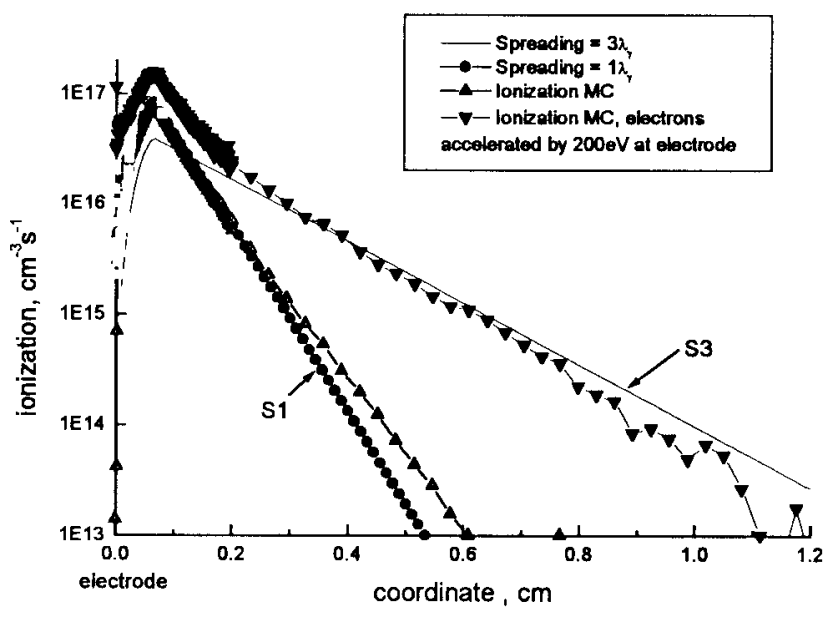

Fig. 5. The $\gamma$ ionization profiles at $j_{0}=20 \mathrm{~mA} / \mathrm{cm}^{2}$, which were calculated by use of Monte-Carlo technique (the corresponding curve is marked by "MC") and by fast modeling for $k=1,3$, are shown jointly.

$\gamma$ electrons is accounted for, the ion flux at $|x|<L_{p}$ is controlled by $\gamma$ ionization, even in the plasma bulk outside of the sheath. From (9) we can estimate the nonlocal ionization at $|x|<L_{p}$ as $I_{0} \exp \left(-\left|x \mp L_{p}\right| /\left(k \lambda_{\gamma}\right)\right)$, where $I_{0}=\int_{L_{p}}^{L_{0}} I_{\gamma}(x) \exp \left[-\left(x-L_{p}\right) / k \lambda_{\gamma}\right] d x /\left(k \lambda_{\gamma}\right)$, and “-" corresponds to the right part of the discharge, "+" to the left one. Integration of (9) with boundary condition $\delta(d n / d x)=n$ at $x=L_{p}$ yields the following value of the plasma density at the discharge center

$$
n(0)=I_{0}\left(k \lambda_{\gamma}\right)\left(\delta+\left(k \lambda_{\gamma}\right)\right) / D_{a m b} .
$$

Equation (9) gives the central density $n(0)$ as a function of $k \lambda_{\gamma}$. From the expression for $I_{0}$ it follows that $I_{0} k \lambda_{\gamma}$ is constant (on $k \lambda_{\gamma}$ ) if $k \lambda_{\gamma} \gg L-L_{p}=L_{s h}$, and $I_{0}$ is constant (on $k \lambda_{\gamma}$ ) if $k \lambda_{\gamma} \ll L_{s h}$. In our case $k \lambda_{\gamma} \gg \delta$, and the plasma density at the discharge center is proportional to the spatial scale of the spreading $n(0) \sim k \lambda_{\gamma}$ for $k \lambda_{\gamma} \gg L_{s h}$ but $n(0) \sim\left(k \lambda_{\gamma}\right)^{2}$ for $k \lambda_{\gamma} \ll L_{s h}$. It is seen from Fig. 4(a) that an increase of the spatial scale of the spreading by a factor of $3\left(k \lambda_{\gamma} \sim L_{s h}\right)$ results in an increased central plasma density by approximately a factor of three as well.

\section{Study of Spatial Profiles of Nonlocal IONIZATION, PRODUCED By GAMMA ElECTRONS}

At the $\alpha-\gamma$ transition, the sheath thickness automatically shrinks to a length of the order of several mean free paths of gamma electrons [10]; see Fig. 3(b). This implies that the ionization in the $\gamma$ regime is essentially nonlocal for any pressure and the local approximation fails even at very high pressures.

To verify our simplified model for the ionization rate (8) we performed a calculation of the $\gamma$ ionization rate by the use of MCC technique. The calculation was performed for a given electric field obtained in a self-consistent fast modeling simulation. The $\gamma$ ionization rate profiles are shown in Fig. 5 . It is seen that at $0<x<0.7 \mathrm{~cm}$ the real form of the $\gamma$ ionization profile from the MCC calculation is very close to the model profile (8) with $k=1$. The typical relaxation length of ionization $\lambda$ (the length after which ionization drops by a factor of $e$ inside the plasma) depends on the energy of the electrons, as can be seen in Fig. 5. If the energy of secondary electrons at the cathode is artificially increased up to 200 $\mathrm{eV}$, the characteristic decay length of ionization is increased approximately by a factor of three.

The electron Boltzmann equation has to be solved in order to investigate the dependence of $k \lambda_{\gamma}$ on electron energy. We focus on the study of the decay of ionization inside the low field plasma. We assume that the energetic electrons are injected at $x=0$ (plasma-sheath boundary) with a flux distribution $\Gamma(\varepsilon)$. This corresponds to the negative glow region of $\mathrm{rf}$ and dc discharges. The secondary electrons lose their energy in inelastic (excitation and ionization) collisions and propagate inside the discharge. For the investigation of $\gamma$ electron decay in a plasma, we neglect their backward losses to the wall, since the large sheath potential traps electrons. If the energy of the $\gamma$ electron is much higher than the ionization threshold, the inelastic collision integral can be simplified

$$
\begin{aligned}
S t^{*}(f) & =\sum_{k}\left[\nu_{k}\left(\varepsilon+\varepsilon_{k}\right) f\left(\varepsilon+\varepsilon_{k}\right)-\nu_{k}(\varepsilon) f(\varepsilon)\right] \\
& \approx \frac{\partial}{\partial \varepsilon}\left(V_{\varepsilon} f(\varepsilon)\right), \quad V_{\varepsilon} \equiv \sum_{k}\left[\varepsilon_{k} \nu_{k}(\varepsilon)\right] .
\end{aligned}
$$

For light gases like hydrogen the elastic scattering crosssection is comparable with the inelastic one. This assumption enables the authors of [13] to assume that the beam of $\gamma$ electrons propagates without scattering. Energetic electrons generate progeny electrons with lower energies [14]. So secondary electrons are not capable of further ionization and can be excluded from consideration. Thus, the kinetic equation for the $\gamma$ electrons in light gases $(\mathrm{H}, \mathrm{He})$ reads

$$
v_{x} \frac{\partial f}{\partial x}-\frac{\partial V_{\varepsilon} f}{\partial \varepsilon}=\Gamma(\varepsilon) \delta(x), \quad v_{x}=\sqrt{2 \varepsilon / m} .
$$

Equation (11) can be easily integrated along characteristics

$$
x=\int_{\varepsilon}^{\varepsilon_{0}} \frac{v_{x}}{V_{\varepsilon}} d \varepsilon, \quad f(x, \varepsilon)=\frac{\Gamma\left(\varepsilon_{0}\right)}{v_{x}\left(\varepsilon_{0}\right)} .
$$

The characteristic scale of beam energy and ionization decay is $k \lambda_{\gamma} \sim v_{x} \varepsilon / V_{\varepsilon}$. If we determine the cost of ionization as $\varepsilon_{c i} \sim V_{\varepsilon} / \nu_{i}$, then $k \lambda_{\gamma} \sim \lambda_{i}\left(\varepsilon / \varepsilon_{c i}\right)$ is the electron mean free path for ionization multiplied by a ratio of the beam energy to the cost of ionization.

In cases where atoms have a large number of electrons in the electron shell $\left(Z_{e l}\right)$, the elastic collision frequency is larger than the inelastic one (their ratio scales as $Z_{e l}$ at high energies [13]). Under these conditions one can try to assume that the EDF is close to isotropic, due to fast pitch-angle scattering. In this case, the kinetic equation reads

$$
-D_{x} \frac{\partial^{2} f}{\partial x^{2}}-\frac{\partial V_{\varepsilon} f}{\partial \varepsilon}=\Gamma(\varepsilon) \delta(x)
$$

where the total density of $\gamma$ electrons $n_{\gamma}=\int f d \varepsilon$. If $V_{\varepsilon}$ can be set to a constant, (12) can be easily solved by Laplace transformation. But the solution can be found by analogy with the nonstationary diffusion problem: if we change variable energy to "time" $\varepsilon_{\max }-\varepsilon \rightarrow t$, where $\varepsilon_{\max }$ is the maximum 
energy of the injected electrons, we see that (12) is a diffusion problem in time with well-studied solutions.

First we analyze the case when a mono-energetic beam of electrons is injected: $\Gamma(\varepsilon)=\Gamma_{0} \delta\left(\varepsilon_{\max }-\varepsilon\right)$, where $\Gamma_{0}$ is the total flux of gamma electrons. The solution of (12) is

$$
f(\varepsilon, x)=\frac{\Gamma_{0} \exp \left(-\frac{V_{\varepsilon} x^{2}}{4 D_{x}\left(\varepsilon_{\max }-\varepsilon\right)}\right)}{2 \sqrt{\pi V_{\varepsilon} D_{x}\left(\varepsilon_{\max }-\varepsilon\right)}} .
$$

Normalizing $x$ with $2 \sqrt{D_{x} \varepsilon_{\max } / V_{\varepsilon}}$, one can see that at $x>1$ the EDF decreases rapidly as $\exp \left(-x^{2}\right)$. The ionization produced by $\gamma$ electrons is given by $I(x)=\int \nu_{i} f(\varepsilon) d \varepsilon$. For our approximation of constant collision frequency, the ionization rate is simply proportional to the integral over $f$, the density of gamma electrons. Integrating function (13), one can find that the ionization profile is well described by a function

$$
G(x)=\frac{2 \exp \left(-x^{2}\right)}{x^{2}+1} .
$$

As we can see the ionization decay is much faster than exponential with a typical scale $2 \sqrt{D_{x} \varepsilon_{\max } / V_{\varepsilon}} \equiv$ $2 \sqrt{D_{x} \varepsilon_{\max } / \nu_{i} \varepsilon_{c}}$. A similar estimate was found in [2]. In case of a uniform flux of $\gamma$ electrons the EDF is

$$
f(\varepsilon, x)=\int_{\varepsilon}^{1} \exp \left[-x^{2} /(1-\varepsilon)\right] / \sqrt{(1-\varepsilon)} d \varepsilon
$$

and the ionization rate decreases very rapidly in a manner similar to the previous case as $G(x)=\exp \left(-x^{2}\right) /\left(x^{4}+1\right)$. So in both cases a Gaussian drop of ionization should be expected.

The generalization of the solution (13) is possible for arbitrary functions $V_{\varepsilon}, D_{x}$ of energy

$$
f(\varepsilon, x)=\frac{1}{2 V_{\varepsilon} \sqrt{t_{\mathrm{eff}}}} \Gamma_{0} \exp \left(-x^{2} / 4 t_{\mathrm{eff}}\right)
$$

where

$$
t_{\mathrm{eff}}=\int_{\varepsilon}^{\varepsilon_{\max }} \frac{D_{x}(\varepsilon)}{V_{\varepsilon}(\varepsilon)} d \varepsilon
$$

We performed the Monte Carlo simulation for comparison with the analytical formula, the cross-section set being taken from [12]. An electron beam with initial energy of $200 \mathrm{eV}$ was injected into the argon gas at a pressure of 3 torr. The EDF of the electron beam becomes close to isotropic after passing a distance of the order of several mean free paths (which is about $0.1 \mathrm{~mm}$ ) as can be seen from Fig. 6. The distribution functions and ionization profiles are shown in Figs. 6 and 7, respectively. One can see that the ionization profile is close to Gaussian with a scale of $2.4 \mathrm{~mm}$. The estimate of the relaxation length at energy $100 \mathrm{eV}, 2 \sqrt{D_{x} \varepsilon_{\max } / V_{\varepsilon}}$ is $2.2 \mathrm{~mm}$. When calculating the relaxation length, one needs to account for the anisotropic form of the elastic scattering cross sections for a correct estimation of the diffusion coefficient.

The EDF's calculated by MCC as a function of energy are compared with the analytical estimate (14) in Fig. 8. One can see reasonable agreement in EDF's too. At lower energies $(\varepsilon<40 \mathrm{eV})$ the MCC EDF is much larger due to the contribution of progeny electrons not included in the analytical model.
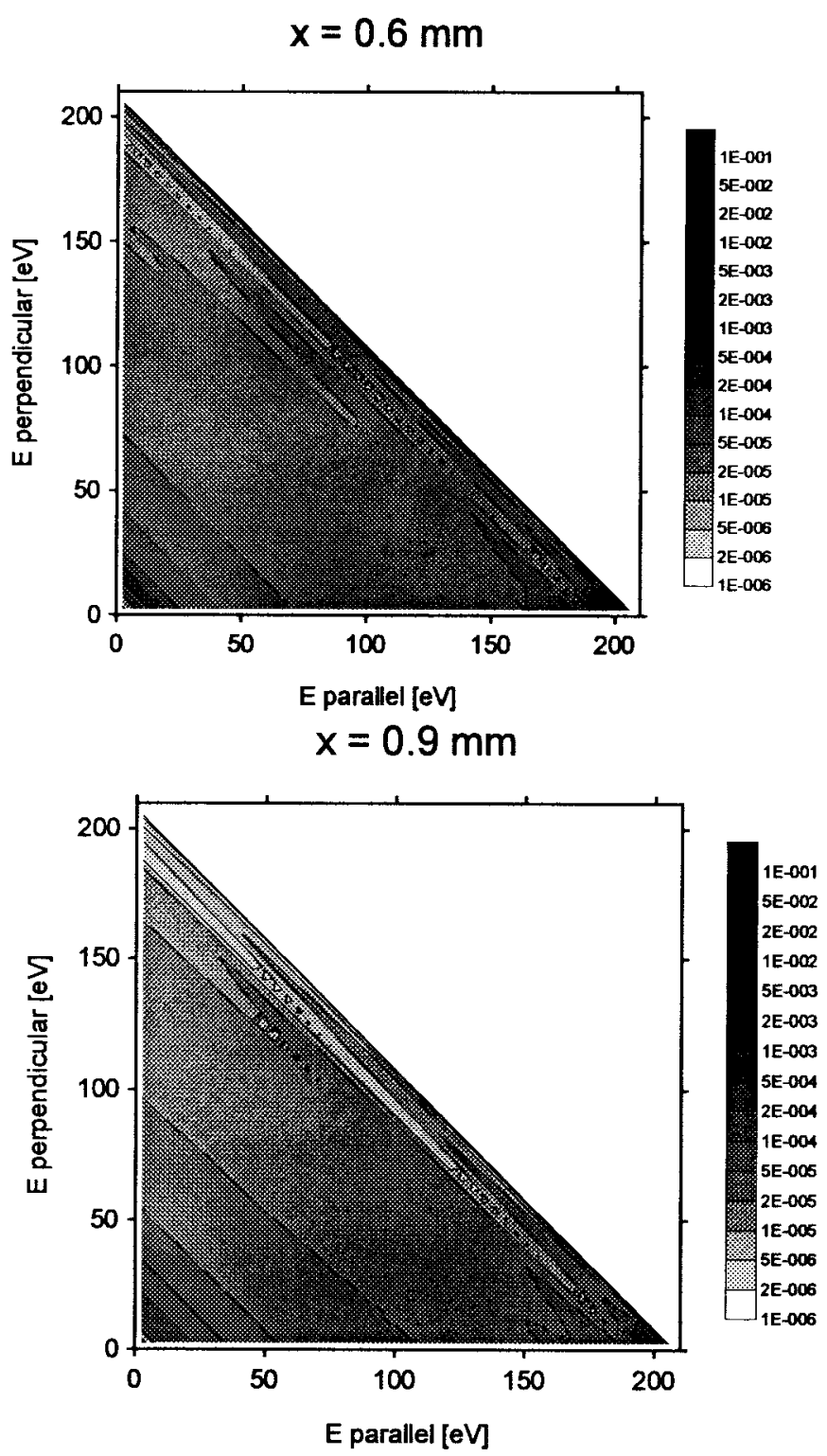

Fig. 6. Monte Carlo calculation of the energy distribution function (EDF) of gamma electrons injected into argon at argon pressure 3 torr, at $x=0$ as a mono-energetic beam with energy of $200 \mathrm{eV}$.

\section{CONCLUSIONS}

The model considered for fast simulation of a radiofrequency capacitively coupled discharge at intermediate pressures allows us to evaluate the discharge parameters in detail even when the local approach for $\gamma$ electrons is not valid. It was shown that the non locality of $\gamma$ electrons is the reason for the steep increase of the central plasma density in the $\gamma$ regime.

The present study demonstrates that in the $\gamma$ regime the central plasma density at the discharge center is proportional to the spatial scale of nonlocality.

To estimate this spatial scale the electron Boltzmann kinetic equation was solved analytically. The analytical results are consistent with ionization profiles and EDF's calculated by the use of MCC. 


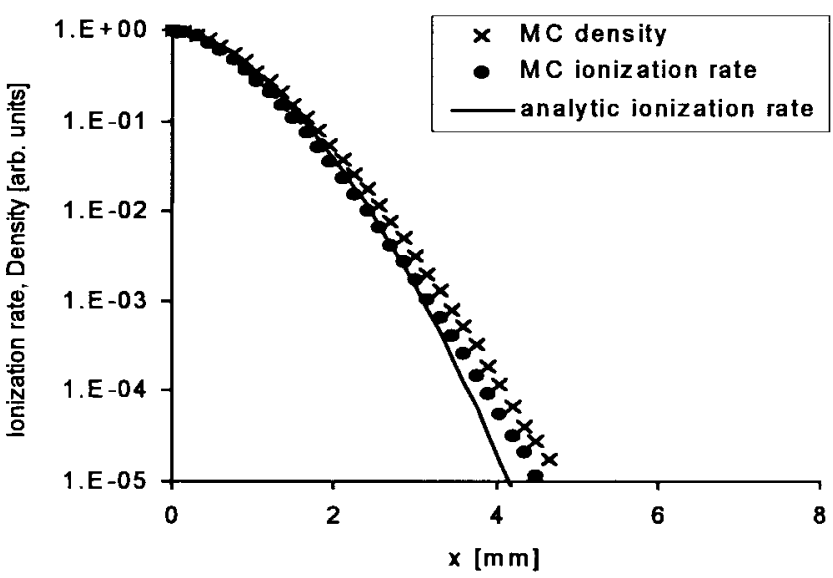

Fig. 7. Ionization profile (filled circles), and density of fast electrons (crosses) of an electron beam with initial energy of $200 \mathrm{eV}$ in argon (3 torr), calculated by use of Monte Carlo technique. The solid line represents the analytical estimate for ionization: $G(x)=\exp \left(-(x / 2.4 \mathrm{~mm})^{2}\right)$ / $(x / 2.4 \mathrm{~mm})^{2}+1$.

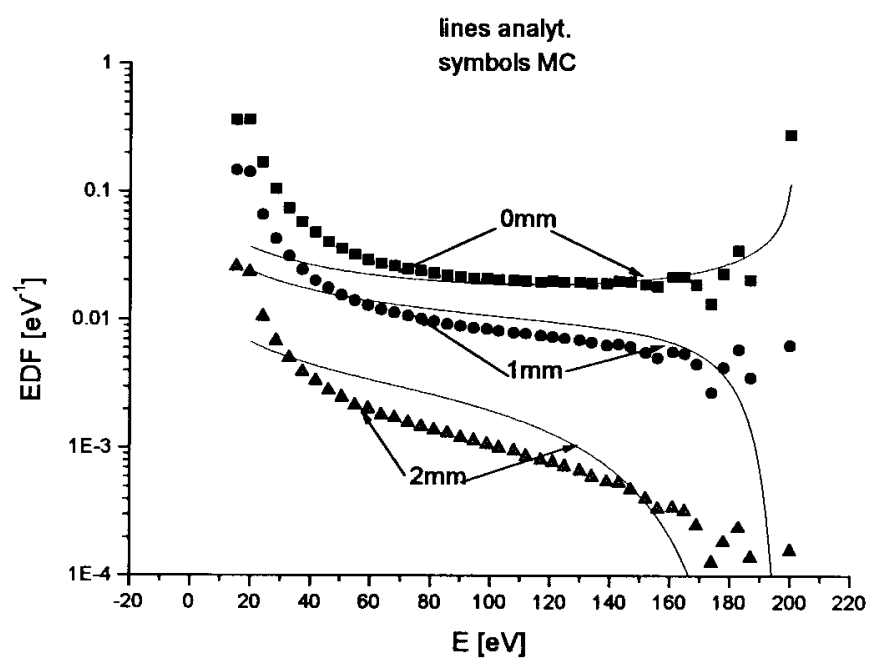

Fig. 8. The EDF's for a mono-energetic beam of electrons $200 \mathrm{eV}$ were calculated by use of Monte Carlo technique (solid lines). The lines with symbols represent analytical estimate for EDF's (14).

The good agreement should encourage the further investigation of EDF relaxation in more complicated situations.

\section{ACKNOWLEDGMENT}

The authors would like to thank B. Ramamurthi and Profs. L. Tsendin, U. Korsthagen, and D. Economou are gratefully acknowledged.

\section{REFERENCES}

[1] Y. P. Raizer, M. N. Schneider, and N. A. Yatsenko, Radio-Frequency Capacitive Discharges. Boca Raton, FL: CRC Press, 1995, p. 292.

[2] V. A. Godyak and A. S. Khanneh, "Ion bombardment secondary electron maintenance of steady RF discharge," IEEE Trans. Plasma Sci., vol. PS-14, pp. 112-123, 1986.

[3] A. Bogaerts, M. van Straaten, and R. Gijbels, "Monte Carlo simulation of an analytical glow discharge: Motion of electrons, ions and fast neutrals in the cathode dark space," Spectrochim. Acta Part B, vol. 50, pp. 179-196, 1995 .
[4] V. Godyak and R. Piejak, "Measurements of electron energy distribution functions in low pressure RF discharges," Plasma Sources Sci. Technol., vol. 1, p. 36, 1992.

[5] L. D. Tsendin, "Electron motion and averaged field in electrode sheathes of RF discharge," Sov. Phys.-Tech. Phys., vol. 35, no. 8, pp. 53-58, 1990.

[6] A. S. Smirnov and L. D. Tsendin, "The space-time-averaging procedure and modeling of the RF discharge," IEEE Trans. Plasma Sci., vol. 19, pp. $130-140,1991$.

[7] L. D. Tsendin, "Electron distribution in a weakly ionized plasma in an inhomogeneous electric field," Sov. J. Plasma Phys., vol. 8, no. 2, pp. 228-233, 1982

[8] S. V. Berezhnoi, I. D. Kaganovich, and L. D. Tsendin, "Fast modeling of low pressure radio-frequency collisional capacitively coupled discharge and investigation of nonMaxwellian electron distribution function," Plasma Sources Sci. Technol., vol. 7, no. 3, pp. 268-281, 1998.

[9] I. D. Kaganovich, L. D. Tsendin, and N. A. Yatsenko, "Two dimensional RF discharge at the medium pressures," Sov. Phys.-Tech. Phys., vol. 39, pp. 1215-1226, 1994.

[10] Y. P. Raizer, Gas Discharge Physics. Berlin, Germany: SpringerVerlag, 1991, p. 449

[11] N. B. Kolokolov and A. A. Kudryavtsev, "Interaction processes with creation of fast electrons in the low temperature plasma," Physica Scripta, vol. 50, pp. 371-402, 1994

[12] A. Bogaerts and R. Gijbels, "Modeling of metastable argon atoms in a direct-current glow discharge," Phys. Rev. A, vol. 52, no. 5, pp. 3743-3751, 1995.

[13] V. I. Kolobov and L. D. Tsendin, "Analytic model of the cathode region of a short glow discharge in light gases," Phys. Rev. A, vol. 46, no. 12, pp. 7837-7851, 1992.

[14] L. R. Peterson and J. E. Allen, "Electron impact cross section for argon," J. Chem. Phys., vol. 56, no. 12, p. 6068, 1971.

[15] C. B. Opal, W. K. Peterson, and E. C. Beaty, "Measurements of secondary-electron spectra produced by electron impact ionization of a number of simple gases," J. Chem. Phys., vol. 55, no. 8, p. 4100, 1971.

[16] M. Surendra, D. B. Graves, and I. J. Morey, "Electron heating in low pressure RF glow discharge," Appl. Phys. Lett., vol. 56, no. 11, pp. 1022-1024, 1990.

Stanislav V. Berezhnoi received the Ph.D. degree in physics from the Ioffe Physical-Technical Institute in 1999.

He has been working at St. Petersburg Technical University, Russia, as a postdoctoral Researcher. His research interests are in plasma processing discharges and implementation of methods of fast and effective plasma modeling.

Igor D. Kaganovich received the Ph.D. degree in physics from the Ioffe Physical-Technical Institute in 1992.

He has been working at St. Petersburg Technical University, Russia, since 1996. In 1996-1998, he had an Alexander von Humboldt fellowship and worked at the University of Bochum, Germany. Currently, he is a Research Associate at the University of Houston, TX. His research interests are in plasma processing discharges, development of semi-analytical methods of fast and effective plasma modeling, kinetic theory of plasma and gases, physics of nonlinear systems, pattern formation in plasma physics, and modeling of stochastic systems.

Martin Mišina was born in Olomouc, Czech Republic, in 1966. He received the Ph.D. degree in physics from the Charles University of Prague, Czech Republic, in 1996.

His main research interests lie within sputter deposition technology including development of sputter systems with enhanced ionization, magnetron deposition of nano-composite thin films, and computer simulation and modeling of magnetron plasma. He is currently a Junior Scientist at the Institute of Physics, Academy of Sciences of the Czech Republic, Prague. 
Annemie Bogaerts was born in Belgium on October 25, 1971. She received the M.S. degree in chemistry and the Ph.D. degree in science from the University of Antwerp, Belgium, in 1993 and 1996, respectively.

She is now working as a postdoctoral Researcher at the University of Antwerp. Her current research interest is in the modeling of glow discharge plasmas, as well as in experimental plasma diagnostics to validate the modeling results.
Renaat Gijbels was born in Belgium on January 1, 1939. He received the Ph.D. degree in science from the University of Ghent, Belgium, in 1965.

He became a Professor of Physical Chemistry at the University of Antwerp, Belgium, in 1973. His research interests are in micro, surface, and trace analysis by electron, ion and laser beam techniques: fundamentals, methodology, and applications. 